\title{
E- Health Analysis Element for Supporting Therapeutic through Ancient Indian Medical Science
}

\author{
Dr. G. M. Kadhar Nawaz \\ Director, MCA, \\ Sona College of Technology, \\ Salem- 5
}

\author{
S. Lakshmi, \\ Lecturer, MCA, \\ Sona College of Technology, \\ Salem- 5
}

\author{
JayaSudha \\ Lecturer, MCA, \\ Sona College of Technology \\ Salem- 5
}

\begin{abstract}
:
Very recently there has been a drastic difference in people's demands and expectations of health care systems. A large quantity of information is easily available through the internet, which leads to increased patient knowledge. As the time is passing by, the electronic devices are shrinking in size and price. Taking the advantage of this advanced technology, our demands are leading to health care that improve quality of life throughout the continuum of life.. People are turning towards the Indian culture and are trying to adapt it into their day today life from the ancient Indian medical sciences for their par in excellence, when compared to the modern allopathic treatment. Health care monitoring can now be done ubiquitously and there are several different research projects into wearable health monitoring. However, all the projects are at a prototype stage and not in wide scale use. Our work is concentrated on creating a wearable E- Health analysis element, which will support the medical science of Swayam Nadi Parikshan Various diseases can be controlled by Swayam Nadi Parikshan where by the use of it a transition state of health is maintained from a diseased one. This paper presents a prototype of a non-invasive wellness monitoring system, capable of recording and analyzing continuous pulse rate, heart beat etc... data received from the human body. This system provides an application for recording activities, events and potentially important medical symptoms. The collected data are taken for further diagnosis. This wearable EHealth analysis element helps the patient and the medical practitioner to be well informed about the health condition of the patients. In times of abnormality an alert is sent to both the wearer and the consultant via his personal digital assistant (PDA), for necessary action to be taken.
\end{abstract}

The full text of the article is not available in the cache. Kindly refer the IJCA digital library at www.ijcaonline.org for the complete article. In case, you face problems while downloading the full-text, please send a mail to editor at editor@ijcaonline.org 